\title{
Update Ersttrimesterscreening - was ist neu in 2015?
}

\author{
Stefan Verlohren
}

\begin{tabular}{|c|c|c|c|}
\hline \multicolumn{4}{|l|}{ Übersicht } \\
\hline Einleitung & 243 & Ersttrimesterscreening & \\
\hline Ersttrimesterscreening auf & & auf Präeklampsie & 248 \\
\hline Chromosomenstörungen & 243 & Ausblick & 251 \\
\hline $\begin{array}{l}\text { Ersttrimesterscreening } \\
\text { auf nichtchromosomale }\end{array}$ & & & \\
\hline fetale Fehlbildungen & 247 & & \\
\hline
\end{tabular}

\section{Einleitung}

Das Ersttrimesterscreening (ETS), klassischerweise in $11+0$ bis $13+6$ Schwangerschaftswochen (SSW) durchgeführt, hat sich in den vergangenen Jahren zu einer Schlüsseluntersuchung in der Schwangerschaft entwickelt. Das von Kypros Nicolaides aus London entwickelte Konzept der „Umkehr der Pyramide der Schwangerenvorsorge“ ist vielerorts mittlerweile akzeptiert und in die Praxis umgesetzt. Laut diesem Konzept ist es möglich, bereits im 1 . Trimester eine Vielzahl von möglichen Schwangerschaftskomplikationen vorherzusagen und die weitere Schwangerschaft zielgerichtet zu planen. Frauen, die ein hohes Risiko für z.B. Präeklampsie aufweisen, können entsprechend engmaschiger überwacht werden, und es kann frühzeitig präventiv vorgegangen werden. Bei niedrigem Risiko können die Überwachungsintervalle großzügiger sein. Andererseits erfährt das Ersttrimesterscreening auf Aneuploidien durch die flächendeckende Verfügbarkeit der nichtinvasiven Chromosomendiagnostik (non invasive prenatal test, NIPT) einen grundlegenden Wandel.

Die nichtinvasive Chromosomendiagnostik hat das Ersttrimesterscreening revolutioniert.

War das Screening auf Chromosomenstörungen bisher streng die Domäne des Ultraschalls und der ultraschallgestützten Interventionen, stellt die Möglichkeit, die
Anzahl der Chromosomen des Feten aus dem Blut der Mutter zu bestimmen, eine Herausforderung für die Pränataldiagnostiker dar. Hier müssen sowohl Antworten auf den Stellenwert als auch auf die ethischen Herausforderungen der neuen Screeningmethode gefunden werden.

Der vorliegende Artikel fasst die aktuellen Entwicklungen aus dem Bereich des Ersttrimesterscreenings zusammen. Der Schwerpunkt liegt hierbei auf den Neuerungen rund um den NIPT, das Präeklampsiescreening sowie das Screening auf nichtchromosomale fetale Fehlbildungen.

\section{Ersttrimesterscreening auf Chromosomenstörungen}

\section{Nichtinvasive Pränataldiagnostik - eine neue Ära des Screenings auf Chromosomenstörungen}

Seit im Jahr 1997 entdeckt wurde, dass zellfreie fetale DNA (cell free fetal DNA, cffDNA) im Blut der Mutter zirkuliert, wurde diese Methode konsequent für die Pränataldiagnostik nutzbar gemacht [1]. Zuerst basierte die Technik auf der Sequenzierung und Quantifizierung des Gens Placenta-specific 4 (PLAC4) im Blut der Mutter. Dieses Gen, das auf Chromosom 21 liegt, ist nur in der Plazenta exprimiert und somit fetaler Abstam- 
mung. Bei Detektion zweier separater Allele in einem 1:1-Verhältnis konnte somit auf Euploidie geschlossen werden, während bei 2:1 auf die Duplikation eines der beiden Allele und somit Trisomie 21 geschlossen werden konnte [2,3]. Diese Vorgehensweise erwies sich jedoch als fehleranfällig und aufwendig.

Durch die Verbesserung der Sequenziertechnik und die Verfügbarkeit von Hochdurchsatzsequenzierung, dem „Next Generation Sequencing“ (NGS), war es möglich, die gesamte im maternalen Plasma zirkulierende DNA zu amplifizieren und sequenzieren. Im Gegensatz zur Sequenzierung nur eines Gens auf dem Chromosom 21 können mit NGS kosteneffektiv und schnell große DNA-Mengen vervielfältigt werden. Hier gibt es verschiedene Herangehensweisen:

- „Massively parallel Sequencing“ oder „Whole-genome Approach“

- „Targeted Approach“

- „Singe Nucleotid Polymorphisms“, SNP

Beim „Massively parallel Sequencing“ oder „Wholegenome Approach“ wird das gesamte zirkulierende DNA-Material sequenziert und mit einem Referenzgenom verglichen [4]. Dieses Verfahren nutzt beispielsweise der Praena-Test ${ }^{\circledR}$ von Lifecodexx. Alternativ werden beim „Targeted Approach“ einzelne DNA-Zielfragmente amplifiziert und sequenziert. Dieses Verfahren nutzt beispielsweise der Harmony-Test ${ }^{\circledR}$ von Ariosa/Roche [5]. Schließlich wendet ein 3. Verfahren die Sequenzierung von Varianzen in einem bestimmten Genlocus, die sog. Einzelnukleotidpolymorphismen (Singe Nucleotid Polymorphisms, SNP) an. Diese Technik liegt beispielsweise dem Panorama ${ }^{\circledR}$-Test von Natera zugrunde.

\section{Aussagekraft von NIPT für Trisomie 21}

In der Beratungssituation kann man die Aussagekraft von NIPT für Trisomie 21 vereinfacht wie folgt darstellen: Ausgehend von einer Population von 100000 Schwangerschaften bedeuten eine Detektionsrate von $99 \%$ und eine Falschpositiv-Rate von $0,1 \%$, dass von diesen 100000 Schwangerschaften 100 durch ein falsch-positives Ergebnis auffallen werden. Die durchschnittliche, altersunabhängige Prävalenz von Trisomie kann mit 1:500 angegeben werden. Das heißt, dass von den 100000 Schwangerschaften 200 mit Trisomie 21 zu erwarten sind, von denen 198 mit NIPT korrekt detektiert werden (DR 99\%). Gleichzeitig bedeutet es aber auch, dass von den insgesamt 298 testpositiven Schwangerschaften (100 falsch-positive plus 198 richtig-positive) eben nur 198 tatsächlich eine Trisomie 21 haben, was einem positiven prädiktiven Wert von $66 \%$ entspricht [12].
Die Sequenzierverfahren weisen eine vergleichbare Genauigkeit in der Detektion der „großen“ Chromosomenanomalien Trisomie 13, 18, 21 auf.

Einzelne Tests bieten auch die Beurteilung von gonosomalen Chromosomenstörungen oder Triploidien an. Auch bei Zwillingsschwangerschaften kann NIPT angewendet werden.

\section{Leistungsfähigkeit von NIPT}

NIPT ist mittlerweile in der klinischen Routine angekommen. In einer aktuellen Metaanalyse, die alle relevanten Studien zur Testgüte von NIPT der letzten Jahre zusammenfasst, konnte eine kumulative Detektionsrate für Trisomie 21 von 99,2\% bei einer Falsch-positivRate von 0,09\% für Einlingsschwangerschaften erhoben werden [6]. Die Detektionsraten/Falsch-positiv-Raten (FPR) für Trisomie 18 und 13 werden mit 96,3\%/0,13\% und $91 \% / 0,13 \%$ angegeben. Die Entdeckungsraten für geschlechtsgebundene Aneuploidien, wie Monosomie X (Turner-Syndrom), liegen mit 90,3\%/0,21\% und 93\%/ $0,14 \%$ für andere $\mathrm{X}$-chromosomale Aneuploidien niedriger. Diese Daten wurden sowohl für Hochrisiko- als auch Niedrigrisikokollektive (= Screening) erhoben und bestätigt $[5,7]$.

Eine entscheidende Rolle für das Gelingen des Tests und somit für die Interpretierbarkeit der Ergebnisse von NIPT ist der Anteil fetaler DNA an der insgesamt aus dem mütterlichen Blut gewonnenen DNA, die sog. fetale Fraktion (,fetal fraction“). Diese liegt bei 5-15\%, im Mittel bei $10 \%$ und muss mindestens $4 \%$ betragen, um ein interpretierbares Ergebnis zu ermöglichen [8].

In 5\% der Fälle kommt es zu einem nicht interpretierbaren Ergebnis.

Die Gründe für einen geringen Anteil fetaler DNA an der Gesamt-DNA sind neben einem zu geringen Plazentagewicht aufgrund eines zu frühen Testzeitpunkts ein hoher mütterlicher Body-Mass-Index (BMI) $[9,10]$. Auch bei Trisomie 13 und 18 kann aufgrund eines mit den Krankheitsbildern assoziierten geringeren Plazentagewichts ein zu geringer Anteil fetaler Fraktion resultieren [11].

Bei NIPT handelt es sich um einen Screeningtest, das muss in der Beratungssituation deutlich gemacht werden. Auch wenn die berichteten Detektionsraten nah an denen eines diagnostischen Tests liegen, ist im Falle eines positiven Ergebnisses eine Bestätigung durch die 

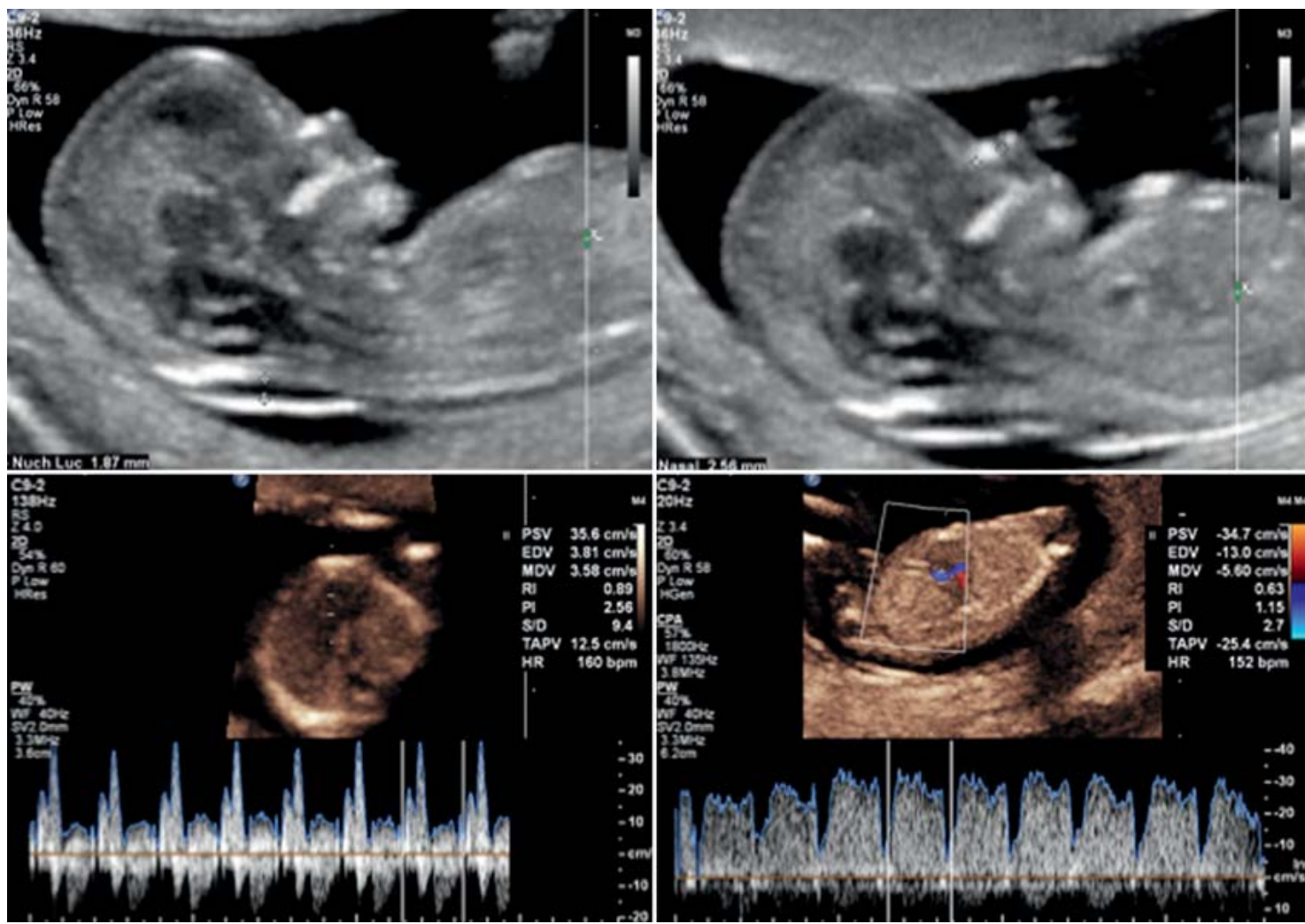

Abb. 1 Sonomarker für Chromosomenstörungen.

invasive Diagnostik (Amniozentese oder Chorionzottenbiopsie) erforderlich. Die Ursache hierfür ist, dass im Falle von Plazentamosaiken falsch positive Ergebnisse resultieren können [13]. Weitere - wenn auch extrem seltene - Fehlerquellen können sein:

- ein früh abgestorbener Kozwilling (dessen DNA auch nach seinem Tod noch von der Plazenta abgegeben wird)

- mütterliche chromosomale Rearrangements oder Mosaike

- ein Malignom mit Sekretion diskordanter DNA [14-16]

\section{Stellenwert von NIPT im Screeningprozess}

Die bisher etablierte Vorgehensweise beim Ersttrimesterscreening auf Chromosomenstörungen war das kombinierte Screening mit Ultraschall und Serumbiochemie. Im Ultraschall werden Hinweiszeichen für Chromosomenstörungen gesucht.

Prominentester Marker im Ultraschall ist die verdickte Nackenfalte (NT).
Auch weitere Ultraschallzeichen wie das fehlende oder dysplastische Nasenbein, ein Rückwärtsfluss im Ductus venosus oder die Trikuspidalklappenregurgitation (Abb. 1) fließen in eine entweder nach Fetal Medicine Foundation (FMF) UK oder Deutschland zertifizierte Risikokalkulation ein $[17,18]$. Weiterhin können noch die Serumparameter PAPP-A und freies Beta-hCG in die Berechnung einbezogen werden. Die hierdurch erreichten Detektionsraten liegen bei ca. $90 \%$ bei einer FPR von 3-5\% [19-21]. Die Ergebnisse werden dann im Rahmen eines individuellen Risikos der Patientin mitgeteilt, in Form von $1: x$. Gemäß dieser Algorithmen kann das individuelle Trisomie-21-Risiko der Patientin in 3 Gruppen eingeteilt werden:

- niedrig

- intermediär

- hoch

Je nach angewendetem Algorithmus sind die Grenzwerte für die Hochrisikogruppe 1:50 (FMF UK) oder $1: 150$ (FMF Deutschland), es folgt die Intermediärrisikogruppe und der Schwellenwert zur Niedrigrisikogruppe beträgt 1:1000 (FMF UK) oder 1:500 (FMF Deutschland). 


\section{Stellenwert von NIPT im ETS}

Wie kann sich NIPT nun sinnvoll in das ETS einpassen? Hier gibt es verschiedene Szenarien, die von Faktoren abhängen wie der zukünftigen Kostenentwicklung sowie Modus und Umfang der Kostenübernahme. In den vergangenen Jahren hat durch rasche Verbesserung der Technik eine Kostensenkung eingesetzt, deren Ende wohl noch nicht erreicht ist. Auch von dieser Entwicklung wird der Stellenwert von NIPT im Screeningprozess abhängen. Aktuell ist NIPT nur als IGeL verfügbar. Verschiedene Kassen übernehmen jedoch bereits jetzt die Kosten auf Sonderantrag. Im Moment wird eine angeregte Diskussion über die Vergütung der Leistungen durch die gesetzlichen Krankenkassen geführt. Im gemeinsamen Bundesausschuss wird NIPT gegenwärtig im Rahmen einer Erprobungsrichtlinie beraten.

Das im Moment von den meisten Meinungsbildnern auf diesem Feld empfohlene Vorgehen ist der Einsatz des NIPT in einem kontingenten Modell. Hier würde als primäres Screening weiterhin die Ultraschalluntersuchung als erweitertes ETS, in Kombination mit oder ohne Serumbiochemie, stehen (Abb. 2).

Kontingentes Modell. Ein Testergebnis im Intermediärbereich würde dann NIPT nach sich ziehen. Nach FMF UK sind etwa 12,5\% der gescreenten Schwangeren in diesem Bereich zu erwarten.

Bei einem hohen Risiko würde direkt eine invasive Diagnostik und damit zweifelsfreie diagnostische Klärung empfohlen, bei einem niedrigen Risiko kein weiterer Test.

In der Hochrisikogruppe wäre es bei Vorliegen entsprechender Auffälligkeiten zu kurz gegriffen, nur die per NIPT erkennbaren Chromosomenstörungen abzu-

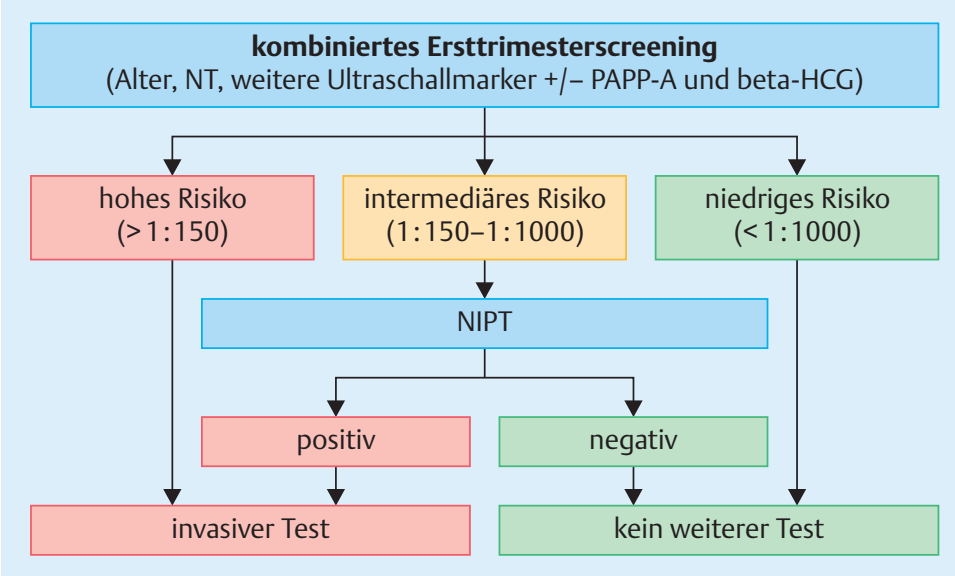

Abb. 2 Flow chart contingent screening NIPT (mod. nach Nikolaides 2014). klären und die Frau bei unauffälligem Ergebnis in falscher Sicherheit zu wiegen. Hinter den Auffälligkeiten können sich auch andere, nicht numerische chromosomale Auffälligkeiten verbergen, die sich per zytogenetischer Karyotypisierung der Array CGH aus dem gewonnenen fetalen Material (Plazentazotten, Fruchtwasser oder Nabelschnurblut) bestimmen lassen. Prinzipiell ist es in Zukunft zwar denkbar, dass ein „Wholegenome Sequencing“ über entsprechende Kostensenkung verfügbar wird; hierfür gibt es aktuell allerdings noch keine Anbieter.

In der Niedrigrisikogruppe scheint NIPT unter den gegenwärtigen Bedingungen ebenfalls nicht angezeigt. Zwar leistet der Test auch im Niedrigrisikokollektiv eine zuverlässige Vorhersage, die Seltenheit der Trisomien 13, 18 und 21 rechtfertigt eine flächendeckende Anwendung (noch) nicht [5].

NIPT empfiehlt sich bei ETS-Ergebnissen im Intermediärrisikobereich und bisher nicht im Hoch- oder Niedrigrisikobereich.

Ausblick. Sollten sich die Kosten soweit reduzieren, dass NIPT als primärer Screeningtest allen Schwangeren angeboten wird, dann ließe sich NIPT dem eigentlichen ETS vorschalten. Eine Folge wäre, dass die entsprechenden Chromosomenstörungen bereits vor der Ultraschalluntersuchung ausgeschlossen wären und sich die Embryosonografie auf die Detektion nichtchromosomaler Fehlbildungen des Feten sowie eine Risikoabschätzung für Schwangerschaftskomplikationen wie Präeklampsie konzentrieren kann.

\section{Tipp für die Praxis}

NIPT kann selbstverständlich auch jederzeit später im Verlauf der Schwangerschaft angewendet werden, bei Erkennen von Hinweiszeichen für Chromosomenstörungen beispielsweise in der Feindiagnostik.

Eine Folge der zunehmenden Anwendung von NIPT ist die Reduktion invasiver Eingriffe. Bereits jetzt, kurze Zeit nach kommerzieller Verfügbarkeit von NIPT, sind die Zahlen der Amniozentesen und Chorionzottenbiopsien deutlich zurückgegangen [22].

Eine zentrale Bedeutung für den betreuenden Frauenarzt kommt der Aufklärung über das Wesen und die Limitationen von NIPT sowie über den Befund zu. Die Aufklärungspflicht ist in Deutschland im Gendiagnostikgesetz geregelt, das immer dann zum Tragen kommt, 
wenn biologisches Material auf die genetischen Eigenschaften der DNA hin untersucht wird.

Die aktuell geführte ethische Debatte über NIPT ist aus Sicht der Pränataldiagnostiker nicht nachvollziehbar. NIPT bringt im Grunde keine grundsätzlich neue Herangehensweise an das Screening auf Chromosomenstörungen mit sich. Lediglich die Art und der Weg der Gewinnung des zu untersuchenden Materials ist eine andere und zwar eine sichere, da das Abortrisiko der invasiven Diagnostik umgangen wird.

\section{Ersttrimesterscreening auf nichtchromosomale fetale Fehlbildungen}

Die zentrale Bedeutung des Ultraschalls für das ETS ergibt sich aus der Möglichkeit, eine Vielzahl von nichtchromosomal bedingten Fehlbildungen zu detektieren. Durch die immer besser werdenden Ultraschallgeräte können in der bereits in der 11.-13. + 6 SSW durchgeführten „Embryosonografie“ viele vormals erst in der Feindiagnostik in der 22. SSW erkannten fetalen Fehlbildungen entdeckt werden. An dieser Stelle sei auf 2 Beispiele näher eingegangen:

- Detektion angeborener Herzfehler

- Spina bifida

Herzfehler. Die fetale Echokardiografie hat sich in den letzten Jahren zunehmend in das 1 . Trimester verlagert. Angeborene Herzfehler stellen mit einer Prävalenz von 3-8/1000 Lebendgeborene eine der häufigsten, klinisch relevanten Fehlbildungen dar [23]. Die Entdeckungsraten von Herzfehlern im Ersttrimesterscreening schwanken. Zwar sind nicht alle Herzfehler bereits im ETS detektierbar, sog. schwere Herzfehler, die kardiologisch-interventionelle und/oder kardiochirurgische Eingriffe erfordern, sind aber in einer Vielzahl von Fällen erkennbar.

Viele Fälle schwerer Herzfehler sind bereits im ETS detektierbar.

Eine große Studie konnte zeigen, dass bei entsprechender Ausbildung gute Entdeckungsraten großer Herzfehler im 1. Trimester erreicht werden konnten (s.Tab. 1). So konnten 50\% der Fälle mit hypoplastischem Linksherz und „Double Outlet right Ventricle“ und 30\% der Fälle mit Transposition der großen Arterien, atrioventrikulärer Septumdefekt, Coarctatio aortae bereits im ETS entdeckt werden [24]. In einer

\section{Tabelle 1}

Verdickte Nackenfalte als Risikofaktor für einen großen Herzfehler (nach [26]).

\begin{tabular}{|lc}
\hline Dicke der Nackenfalte & Prävalenz großer Herzfehler \\
\hline $2,5-3,4 \mathrm{~mm}$ (95. -99. Perzentil) & $1,5 \%$ \\
\hline $3,5-4,4 \mathrm{~mm}$ & $3,3 \%$ \\
\hline $4,5-5,4 \mathrm{~mm}$ & $5,5 \%$ \\
\hline $5,5-6,6 \mathrm{~mm}$ & $15 \%$ \\
\hline $6,5-8,4 \mathrm{~mm}$ & $19 \%$ \\
\hline
\end{tabular}

großen Metaanalyse wurden die Prävalenzen eines großen Herzfehlers bei verdickter NT erhoben (s. Tab. 1) [26].

\section{Tipp für die Praxis}

Eine verdickte Nackenfalte $>95$. Perzentil oder $>$ 2,5 mm stellt einen Risikofaktor für das Vorliegen von Herzfehlern dar, insbesondere, wenn der Chromosomensatz normal ist. Bei normalem Chromosomensatz beträgt die Inzidenz eines großen kardialen Defekts 2,5\%, wenn die NT > 2,5 mm beträgt und 7\% in Feten mit einer NT $\geq 3,5$ mm [25]. Das Risiko für das Vorliegen eines Herzfehlers bei euploiden Feten steigt exponentiell mit der Dicke der NT an.

Neben der NT-Messung haben auch die weiteren Marker des erweiterten ETS eine Rolle als Risikoselektor für kardiale Fehlbildungen wie:

- Nasenbein

- Widerstand im Ductus venosus (DV)

- Trikuspidalklappen-Regurgitation (TR)

Die Kombination von verdickter NT und abnormalem Flussmuster im Ductus venosus bzw. dem Vorliegen einer Trikuspidalklappen-Regurgitation konnte die Entdeckungsraten für Herzfehler bei euploiden Feten steigern. In einer Metaanalyse betrug bei verdickter NT die Sensitivität des abnormalen Ductus-venosus-Flussmusters $83 \%$, bei einer Falsch-positiv-Rate von $20 \%$; bei Feten mit normaler NT betrug die Sensitivität $19 \%$ bei einer Falsch-positiv-Rate von 4\% [27].

Spina bifida. Ein weiteres Beispiel für eine Verlagerung einer bisher hauptsächlich im 2. Trimester diagnostizierten Fehlbildung in das 1 . Trimester ist die Spina 
bifida. Die Detektion von Neuralrohrdefekten ist bereits im 1. Trimester mit einer hohen Entdeckungsrate möglich. Chaoui et al. konnten zeigen, dass die Vermessung der sog. Intracranial Translucency (IT), der sonomorphologischen Darstellung des 4. Ventrikels im mediosagittalen Längsschnitt des Feten, das Vorhandensein einer offenen Spina bifida detektieren kann. In der gleichen Bildeinstellung, die für die Messung der NT verwendet wird, wird der echogene Raum zwischen dem Plexus choroideus des 4. Ventrikels vermessen [28].

Die Sonomorphologie des 4. Ventrikels ermöglicht die Detektion einer Spina bifida bereits im 1. Trimester.

Weitere Parameter, die vermessen werden können und Informationen hinsichtlich eines Neuralrohrdefektes liefern, sind:

- der Hirnstamm (Brain Stem, BS)

- die Cisterna magna (CM)

- der Abstand vom Hirnstamm zum Os occipitale (BSOB)

Eine Abwesenheit oder ein zu schmaler 4. Ventrikel (= zu kleine IT), eine Verschmälerung der CM und eine
Zunahme des BS zeigen das Vorliegen eines Neuralrohrdefektes an (Abb. 3). In einer prospektiven Screeningstudie konnte kürzlich an über 16000 Patientinnen die Reproduzierbarkeit der IT und der anderen Messwerte der hinteren Schädelgrube mit Detektionsraten von bis zu 100\% gezeigt werden [29].

\section{Ersttrimesterscreening auf Präeklampsie}

Im Zuge der „Umkehr der Pyramide der Pränataldiagnostik“ ist in jüngerer Zeit ein weiterer Baustein an Früherkennungsmaßnahmen in diese neue Basis der Pyramide in 11.-13. + 6 SSW eingefügt worden [30]. Die Gruppe um Nicolaides konnte einen Algorithmus etablieren, der es ermöglicht, bereits in diesem Zeitfenster eine suffiziente Vorhersage folgender Risiken zu erhalten:

- Präeklampsie (PE)

- Wachstumsrestriktion

- Notwendigkeit einer vorzeitigen Entbindung aufgrund von PE
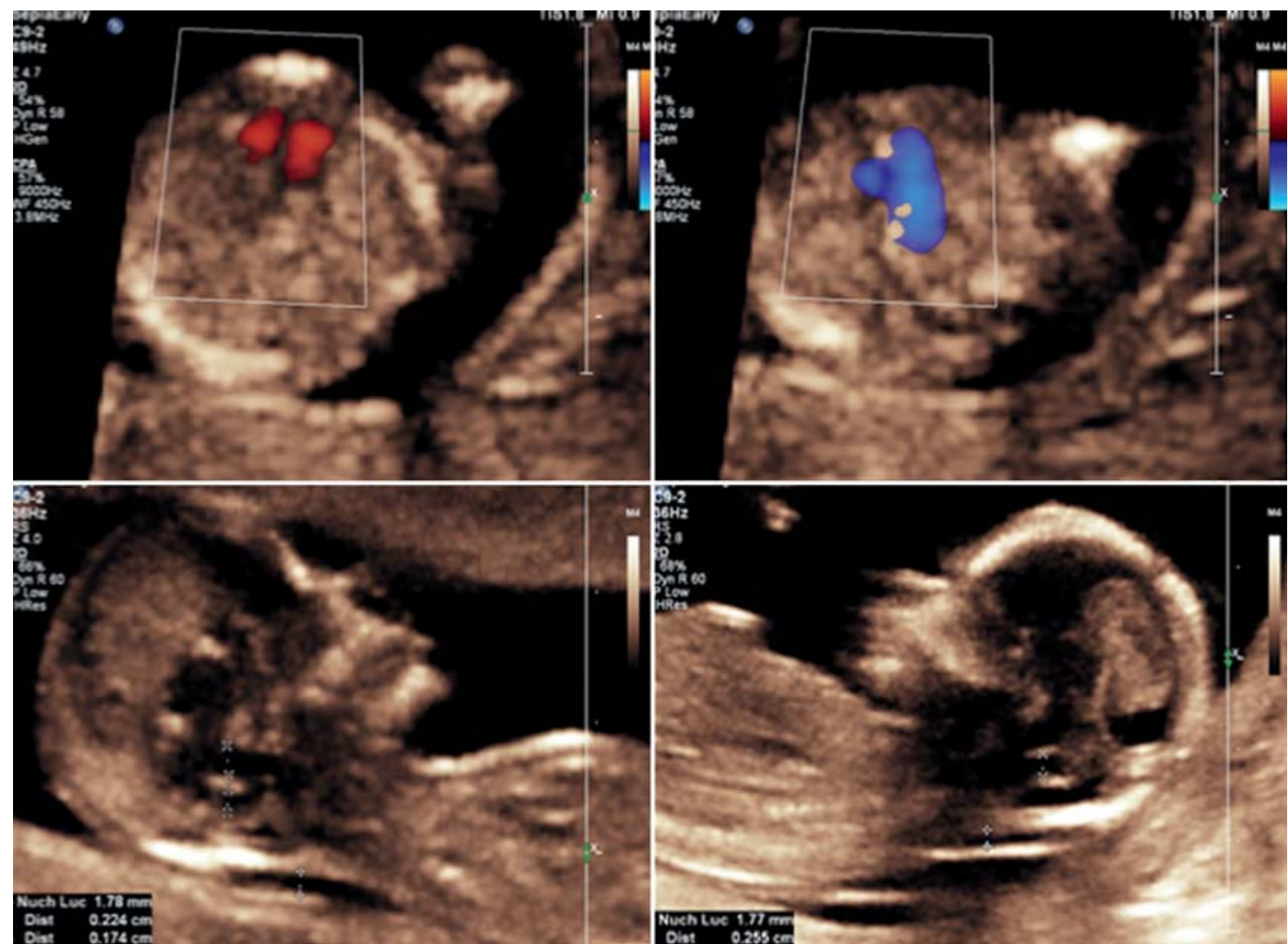

Abb. 3 Ultraschalldiagnostik fetaler Fehlbildungen im 1. Trimester. 


\section{Vorgehen}

Beim Präeklampsiescreening kommt die gleiche prinzipielle Vorgehensweise wie beim Aneuploidiescreening zur Anwendung: Ausgehend von einem A-prioriRisiko, im Wesentlichen der Anamnese der Mutter, werden biophysikalische und biochemische Tests durchgeführt. Die Messwerte werden dann unter Bezugnahme auf Normalwerte für die jeweilige Schwangerschaftswoche in sog. Multiplen des Medians (MoM) ausgedrückt. Ein Algorithmus berechnet aus diesen Messwerten das individuelle Risiko, eine Schwangerschaftskomplikation wie z.B. eine Präeklampsie vor 34 oder vor 37 SSW zu erleiden. Im Falle des PE-Screenings kommen als „biophysikalische“ Werte die Messung des maternalen Blutdrucks und die Doppler-Untersuchung der uterinen Arterien zum Zeitpunkt des ETS zum Einsatz. Die „biochemischen“ Parameter sind das bereits im Aneuploidiescreening angewendete PAPP-A und zusätzlich der plazentare Wachstumsfaktor (PIGF) [31].

\section{Tipp für die Praxis}

Der ETS-Algorithmus zur PE-Detektion kann mittels der Anamnese allein bei einer Falsch-positiv-Rate von $10 \%$ ca. $50 \%$ der Patientinnen erkennen, die im Verlauf der Schwangerschaft eine frühe PE entwickeln. Die Kombination aus Doppler-Sonografie der uterinen Arterien und mütterlichem mittleren arteriellen Blutdruck erbringt bei gleicher FPR eine Detektionsrate von $90 \%$. Die Messung von PAPP-A und PIGF im Serum der Mutter erbringt allein nur eine Detektionsrate von $74 \%$. Kombiniert man nun alle Messwerte, so lassen sich bei einer FPR von $10 \%$ insgesamt $96 \%$ der frühen Präeklampsien detektieren [32].

\section{PE-Prophylaxe}

Acetylsalicylsäure. Ein ETS auf Präeklampsie ist sinnvoll, da mit Aspirin eine Intervention zur Verfügung steht, die eine Reduktion des Auftretens von PE verspricht.

In Metaanalysen der Gruppe um Emmanuel Bujold konnte gezeigt werden, dass der Beginn einer AspirinEinnahme eine bis zu 50\%ige Risikoreduktion zur Folge hat [33]. Diese Daten sind allerdings umstritten, da in den Metaanalysen sehr heterogene Studien mit teils unterschiedlichen Aspirin-Dosierungen und Protokollen verglichen wurden.
Aktuell wird allerdings eine große, durch Mittel der Europäischen Union finanzierte Multizenterstudie durchgeführt, die in einem prospektiv/doppeltverblindeten Studiendesign untersucht, ob Aspirin tatsächlich zu einer geringeren PE-Inzidenz in der Interventionsgruppe führt. In die Studie werden Patientinnen eingeschlossen, die ein positives Screeningergebnis haben (PE-Risiko > 1:50). Diese werden dann randomisiert in eine Gruppe, die $125 \mathrm{mg}$ Aspirin einnimmt, und eine, die ein Placebopräparat erhält. Ergebnisse dieser Studie sind allerdings nicht vor $2017 \mathrm{zu}$ erwarten.

Niedermolekulares Heparin. Eine kürzlich durchgeführte prospektiv randomisierte Studie konnte mit einem anderen hartnäckigen Mythos aufräumen: der angeblichen Wirksamkeit von niedermolekularem Heparin (LMWH), eine Präeklampsie zu verhindern. In der TIPPS-Studie konnte gezeigt werden, dass die Gabe von LMWH nicht geeignet ist, das erneute Auftreten von PE bei Patientinnen zu verhindern, die eine stattgehabte Schwangerschaftskomplikation und eine Thrombophilie haben.

Die aktuelle Datenlage belegt klar die Ineffektivität von LMWH als PE-Prophylaxe [34].

\section{Monitoring nach Risikoabschätzung}

Das ETS auf Präeklampsie stellt eine sinnvolle frühe Vorselektionierung von Patientinnen mit einem hohen Risiko dar. Es ist eine Erweiterung des bisherigen „Screenings“ auf Präeklampsie, das einzig in der Erhebung der Anamnese der Patientin bestand. Viele Patientinnen, die ein scheinbar niedriges Risiko für Präeklampsie hatten, wurden dann durch die Erkrankung zu einem späteren Zeitpunkt überrascht.

Wenn jetzt eine Patientin mit hohem Risiko durch ein positives Screeningergebnis identifiziert wird, kann sie zusätzlich zur präventiven Einnahme von Aspirin engmaschiger überwacht werden. Weiterhin können im Verlauf der Schwangerschaft weitere spezifische PE-Marker, wie beispielsweise der sFlt-1/PlGF-Quotient, bestimmt werden [35]. Patientinnen können so zielgerichtet überwacht und entsprechend individuell betreut werden [36]. 
Kasuistik zum fallorientierten Lernen

\section{Indikation}

Eine 29-jährige Erstgravida/Erstpara stellt sich in 12. + 0 SSW zur Zweitmeinung in der Pränataldiagnostik vor. Extern war im bereits in der 11. $+5 \mathrm{SSW}$ durchgeführten ETS ein fehlendes Nasenbein beschrieben worden. Das Paar ist maximal verunsichert, da es kein Kind mit Chromosomenstörungen möchte. In den 2 Tagen seit der Untersuchung hat sich das Paar intensiv im Internet belesen.

\section{Anamnese}

Größe 167,0 cm, Gewicht vor Schwangerschaft $52,0 \mathrm{~kg}$. Gewicht bei Anamnese 49,0 kg (BMI 17,6). Blutgruppe der Mutter: 0 Rh-pos. Zyklus: regelmäßig, letzte Blutung sicher, Zykluslänge 28 Tage. Konzeption spontan. Bisheriger Schwangerschaftsverlauf unauffällig.

Differenzierter Ultraschall extern erfolgt. Indikation: 1. Trimenon, Nasenbein nicht darstellbar.

\section{Ultraschallbefund}

Ultraschallgerät: EPIQ 7. Untersuchungsmethode: Transabdominalsonografie.

Untersuchungsbedingungen: bedingte Aussage, soweit in dieser SSW beurteilbar. Einlingsschwangerschaft.

Fetale Biometrie (dargestellt zum normalen Mittelwert und 5./95. Perzentile): Herzaktion positiv, Frequenz 156 bpm Scheitelsteißlänge (SSL) $\quad 61,1 \mathrm{~mm}$ Nackentransparenz (NT) $\quad 1,39 \mathrm{~mm}$ biparietaler Durchmesser (BPD) 19,0 mm frontookzipitaler Durchmesser (FOD) Kopfumfang (KU) Abdomenumfang (AU) Femurlänge ( $\mathrm{FL}$ ) $22,0 \mathrm{~mm}$ $64,5 \mathrm{~mm}$ $57,7 \mathrm{~mm}$ $6,7 \mathrm{~mm}$ Nasenbein darstellbar, Länge $\quad 1,9 \mathrm{~mm}$

Sonoanatomie: Schädel gesehen, Herz 4-Kammer-Blick gesehen, Wirbelsäule sonografisch normal, Abdomen sonografisch normal, Magen darstellbar, Blase darstellbar, obere Extremitäten darstellbar, untere Extremitäten darstellbar.
Zusätzliche Marker zur Risikobewertung: Nasenbein darstellbar. Normaler Trikuspidalfluss. Ductus venosus PIV: 0,920.

Chorion frondosum: Hinterwand, Plazentastruktur: unauffällig. Fruchtwasser: unauffällig. Nabelschnur: 3 Gefäße.

Risiko für Trisomien im 1. Trimenon: Trisomie 21 Trisomie 13/18

Hintergrundrisiko: 1:723 1:1322 adjustiertes Risiko: 1:3976 1:5390

Das adjustierte Risiko wurde durch den FMF-Algorithmus 2012 berechnet.

\section{Diagnose}

29-jährige Patientin, Gestationsalter 12. SSW + 0 T. Unauffällige, zeitgerechte Entwicklung, Nasenbein darstellbar (Abb. 4 und 5), unauffällige Nackentransparenz (Abb. 6). Keine Trikuspidalinsuffizienz, Ductus venosus positive A-Welle (Abb. 7). Niedriges adjustiertes Risiko.

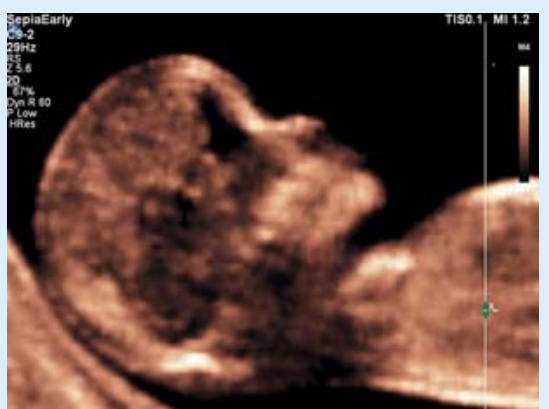

Abb. 4 Darstellung des fetalen Nasenbeins.

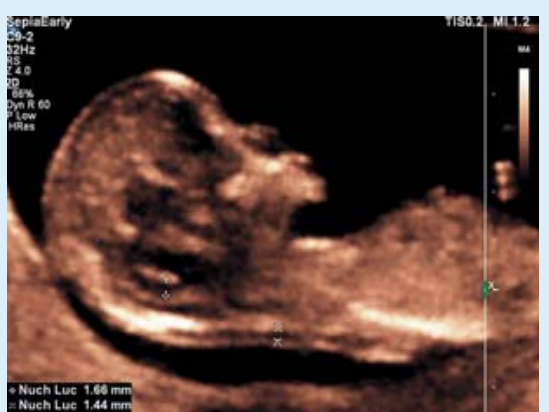

Abb. 6 NT-Messung.

\section{Verlauf}

Das Paar wünschte trotz des niedrigen adjustierten Risikos sowie des nun darstellbaren Nasenbeins eine weitere Abklärung. Es erfolgte die ausführliche Aufklärung über die Optionen der invasiven (CVS sofort oder AC zu einem späteren Zeitpunkt) und nichtinvasiven Diagnostik (NIPT). Das Paar entschied sich für NIPT. Der Test war unauffällig und konnte das Paar beruhigen. Das Paar musste sich nicht dem Risiko eines invasiven Eingriffs aussetzen und hatte einen komplikationslosen weiteren Schwangerschaftsverlauf.

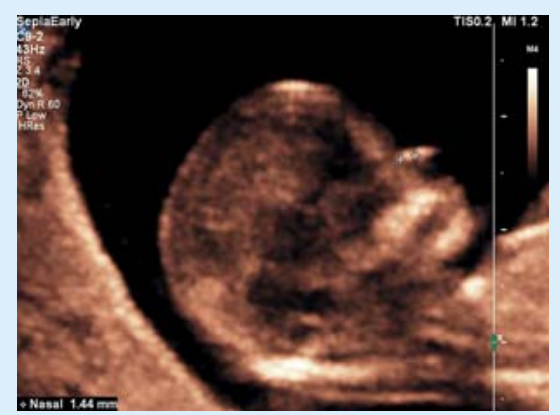

Abb. 5 Biometrie des fetalen Nasenbeins.

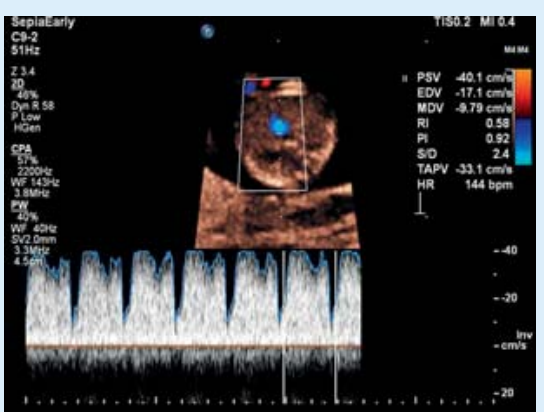

Abb. 7 Flussmessung im Ductus venosus. 


\section{Ausblick}

Der Ultraschall ist und wird die zentrale Komponente des Ersttrimesterscreenings bleiben. Ob NIPT davor als universelles Erstlinienscreening zum Einsatz kommen wird oder ob das bisher durchgeführte gestaffelte Screening mit einem primären Screening per Ultraschall bestehen bleibt, hängt von der weiteren preislichen Entwicklung und auch den gesundheitspolitischen Vorgaben ab. Fest steht, dass aufgrund der Tatsache, dass nur ca. 50\% aller Chromosomenstörungen auf die „großen“ Trisomien 13, 18, 21 entfallen, die Ultraschalldiagnostik, die neben der Embryosonografie auch bereits im 1 . Trimester drohende Schwangerschaftskomplikationen entdecken kann, entscheidend bleibt.

Weiterhin sind nichtchromosomale Fehlbildungen wie Herzfehler oder Spinalkanaldefekte mit immer höherer Sicherheit bereits im 1. Trimester detektierbar. Im PE-Screening kann bereits im ETS auf eine der Erkrankungen getestet werden, die eine Hauptursache mütterlicher und kindlicher Morbidität und Mortalität sind. Hier kann der frühzeitige Beginn einer Aspirin-Prophylaxe tatsächlich auch zu einer substanziellen Reduktion der PE-bedingten Komplikationen beitragen, wenn sich die vielversprechenden Raten zur Risikoreduktion aus den Metaanalysen der Arbeitsgruppe um Bujold auch in der aktuell laufenden prospektiven ASPRE-Studie bestätigen.

Zusätzlich zur Umkehr der Pyramide kommen die zunehmende Individualisierung der Risikovorhersage und dadurch auch maßgeschneiderte Interventionen den Patientinnen zugute.
Perspektivisch ist bei einer weiter fortschreitenden Verbesserung der Sequenzierkapazitäten mit einer Marktreife des Whole-Genome-Sequencing zu rechnen, also der Sequenzierung des gesamten kindlichen Genoms aus einer mütterlichen Blutprobe. Die vor diesem Hintergrund auftretenden ethischen Fragen müssen intensiv diskutiert werden. Wichtig ist aber zum Thema der aktuellen Diskussion festzuhalten, dass die Fragen nicht neu sind, lediglich der Zugang zur fetalen DNA hat sich geändert. Aufgrund des Gendiagnostikgesetzes ist es in Deutschland ärztliche Aufgabe, über das Wesen und die Limitationen dieser Maßnahmen aufzuklären und zu beraten. Hieraus ergibt sich eine besondere Aufgabe für den Pränataldiagnostiker.

\section{Kernaussagen}

Der Ultraschall ist die zentrale Komponente des Ersttrimesterscreenings - und wird es auch weiterhin bleiben. Denn die Ultraschalldiagnostik kann neben der Embryosonografie auch bereits im 1. Trimester drohende Schwangerschaftskomplikationen wie Spina bifida, große Herzfehler oder das Risiko auf Präeklampsie entdecken. Gerade im letzteren Fall kann der risikoadaptierte frühzeitige Beginn einer Aspirin-Prophylaxe zu einer substanziellen Reduktion der PE-bedingten Komplikationen beitragen. Auch wenn die Kosten für NIPT vermutlich weiterhin sinken werden, ist noch offen, ob das Verfahren dem ETS überhaupt vorgeschaltet werden sollte.

Durch das ETS wurde die „Pyramide der Schwangerenvorsorge" umgekehrt und ist vielerorts mittlerweile akzeptiert und in die Praxis umgesetzt. Zusätzlich kommen die zunehmende Individualisierung der Risikovorhersage und dadurch auch maßgeschneiderte Interventionen den Patientinnen zugute. 


\section{Über den Autor}

\section{Stefan Verlohren}

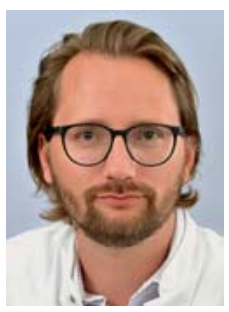

PD Dr. med. Jahrgang 1976. Studium der Humanmedizin in Marburg, Lausanne und Berlin. 2004-2011 Facharztausbildung an der Klinik für Geburtsmedizin und Klinik für Gynäkologie der Charité Berlin. 2005 Promotion. 2007 2009 Forschungsaufenthalt (Post-Doc) am Max-Delbrück-Zentrum für Molekulare Medizin, Berlin. 2011 Facharzt für Gynäkologie und Geburtshilfe. 2012 Habilitation. 2013 Fellowship in Pränataler Diagnostik und Therapie, St. George's Hospital, London. 2014 Schwerpunktbezeichnung Spezielle Geburtshilfe und Perinatalmedizin und DEGUM Stufe II. Seit 11/2014 Facharzt in Oberarztfunktion an der Klinik für Geburtsmedizin der Charité, Berlin. Leiter der Arbeitsgruppe Präeklampsie

\section{Korrespondenzadresse}

PD Dr. med. Stefan Verlohren

Klinik für Geburtsmedizin

Charité Universitätsmedizin, Berlin

Charité Campus Mitte

Charitéplatz 1

10117 Berlin

E-Mail: Stefan.Verlohren@charite.de

\section{Interessenkonflikt}

Berater- und Vortragstätigkeit für Roche Diagnostics und ThermoFisherScientific.

\section{Literatur}

1 Lo YM, Corbetta N, Chamberlain PF et al. Presence of fetal DNA in maternal plasma and serum. Lancet 1997; 350: 485-487

2 Lo YM, Lun FM, Chan KC et al. Digital PCR for the molecular detection of fetal chromosomal aneuploidy. Proc Natl Acad Sci U S A 2007; 104: 13116-13121

3 Everett TR, Chitty LS. Cell-free fetal DNA: the new tool in fetal medicine. Ultrasound Obstet Gynecol 2015; 45: 499-507

4 Fan HC, Blumenfeld YJ, Chitkara U et al. Noninvasive diagnosis of fetal aneuploidy by shotgun sequencing DNA from maternal blood. Proc Natl Acad Sci U S A 2008; 105: 16266-16271

5 Norton ME, Jacobsson B, Swamy GK et al. Cell-free DNA analysis for noninvasive examination of trisomy. N Engl J Med 2015; 372: 1589-1597

6 Gil MM, Quezada MS, Revello R et al. Analysis of cell-free DNA in maternal blood in screening for fetal aneuploidies: updated metaanalysis. Ultrasound Obstet Gynecol 2015; 45: 249-266
7 Boon EM, Faas BH. Benefits and limitations of whole genome versus targeted approaches for noninvasive prenatal testing for fetal aneuploidies. Prenat Diagn 2013; 33: 563 -568

8 Lo YM, Tein MS, Lau TK et al. Quantitative analysis of fetal DNA in maternal plasma and serum: implications for noninvasive prenatal diagnosis. Am J Hum Genet 1998; 62: 768-775

9 Wang E, Batey A, Struble $C$ et al. Gestational age and maternal weight effects on fetal cell-free DNA in maternal plasma. Prenat Diagn 2013; 33: 662-666

10 Ashoor G, Syngelaki A, Poon LCY et al. Fetal fraction in maternal plasma cell-free DNA at 11-13 weeks' gestation: relation to maternal and fetal characteristics. Ultrasound Obstet Gynecol 2012; 41: 26-32

11 Rava RP, Srinivasan A, Sehnert AJ et al. Circulating fetal cell-free DNA fractions differ in autosomal aneuploidies and monosomy $\mathrm{X}$. Clin Chem 2014; 60: 243-250

12 Kagan KO, Eiben B, Kozlowski P. [Combined first trimester screening and cell-free fetal DNA - "next generation screening"]. Ultraschall Med 2014; 35: 229-236

13 Wang J-C, Sahoo T, Schonberg S et al. Discordant noninvasive prenatal testing and cytogenetic results: a study of 109 consecutive cases. Genet Med 2015; 17: 234-236

14 Wang Y, Chen Y, Tian F et al. Maternal mosaicism is a significant contributor to discordant sex chromosomal aneuploidies associated with noninvasive prenatal testing. Clin Chem 2014; 60: 251 259

15 Osborne CM, Hardisty E, Devers P et al. Discordant noninvasive prenatal testing results in a patient subsequently diagnosed with metastatic disease. Prenat Diagn 2013; 33: 609-611

16 Lau TK, jiang FM, Stevenson RJ et al. Secondary findings from noninvasive prenatal testing for common fetal aneuploidies by whole genome sequencing as a clinical service. Prenat Diagn 2013; 33 : $602-608$

17 Nicolaides KH, Spencer K, Avgidou K et al. Multicenter study of first-trimester screening for trisomy 21 in 75821 pregnancies: results and estimation of the potential impact of individual riskorientated two-stage first-trimester screening. Ultrasound Obstet Gynecol 2005; 25: 221-226

18 Merz E, Thode C, Eiben B et al. Individualized correction for maternal weight in calculating the risk of chromosomal abnormalities with first-trimester screening data. Ultraschall Med 2011; 32: 33-39

19 Maiz N, Valencia C, Kagan KO et al. Ductus venosus Doppler in screening for trisomies 21, 18 and 13 and Turner syndrome at $11-13$ weeks of gestation. Ultrasound Obstet Gynecol 2009; 33: $512-517$

20 Kagan KO, Hoopmann M, Abele $\mathrm{H}$ et al. First-trimester combined screening for trisomy 21 with different combinations of placental growth factor, free $\beta$-human chorionic gonadotropin and pregnancy-associated plasma protein-A. Ultrasound Obstet Gynecol 2012; 40: 530-535

21 Kagan KO, Staboulidou I, Cruz J et al. Two-stage first-trimester screening for trisomy 21 by ultrasound assessment and biochemical testing. Ultrasound Obstet Gynecol 2010; 36: 542-547

22 Gil MM, Giunta G, Macalli EA et al. UK NHS pilot study on cell-free DNA testing in screening for fetal trisomies: factors affecting uptake. Ultrasound Obstet Gynecol 2015; 45: 67-73

23 Axt-Fliedner R, Chiriac A, Gembruch U. First and early second trimester fetal heart scanning. Ultraschall Med 2009; 30: 364-375 
24 Syngelaki A, Chelemen T, Dagklis T et al. Challenges in the diagnosis of fetal non-chromosomal abnormalities at 11-13 weeks. Prenat Diagn 2011; 31: 90-102

25 Ghi T, Huggon IC, Zosmer N et al. Incidence of major structural cardiac defects associated with increased nuchal translucency but normal karyotype. Ultrasound Obstet Gynecol 2001; 18: 610 614

26 Clur SA, Ottenkamp J, Bilardo CM. The nuchal translucency and the fetal heart: a literature review. Prenat Diagn 2009; 29: 739 748

27 Papatheodorou SI, Evangelou E, Makrydimas G et al. First-trimester ductus venosus screening for cardiac defects: a meta-analysis. BJOG 2011; 118: 1438-1445

28 Chaoui R, Benoit B, Mitkowska-Wozniak H et al. Assessment of intracranial translucency (IT) in the detection of spina bifida at the 11 -13-week scan. Ultrasound Obstet Gynecol 2009; 34: 249252

29 Chen F, Gerhardt J, Entezami M et al. Detection of spina bifida by first trimester screening - results of the prospective multicenter Berlin IT-Study. Ultraschall Med 2015; [Epub ahead of print]

30 Nicolaides $\mathrm{KH}$. Turning the pyramid of prenatal care. Fetal Diagn Ther 2011; 29: 183-196
31 Wright D, Akolekar R, Syngelaki A et al. A competing risks model in early screening for preeclampsia. Fetal Diagn Ther 2012; 32: $171-178$

32 Akolekar R, Syngelaki A, Poon L et al. Competing risks model in early screening for preeclampsia by biophysical and biochemical markers. Fetal Diagn Ther 2013; 33: 8-15

33 Bujold E, Roberge S, Lacasse Y et al. Prevention of preeclampsia and intrauterine growth restriction with aspirin started in early pregnancy: a meta-analysis. Obstet Gynecol 2010; 116: 402-414

34 Rodger MA, Hague WM, Kingdom J et al.; TIPPS Investigators. Antepartum dalteparin versus no antepartum dalteparin for the prevention of pregnancy complications in pregnant women with thrombophilia (TIPPS): a multinational open-label randomised trial. Lancet 2014; 384: 1673-1683

35 Verlohren S, Herraiz I, Lapaire O et al. New gestational phase-specific cutoff values for the use of the soluble fms-like tyrosine kinase-1/placental growth factor ratio as a diagnostic test for preeclampsia. Hypertension 2014; 63: 346-352

36 Stepan H, Herraiz I, Schlembach D et al. Implementation of the sFlt-1/PIGF ratio for prediction and diagnosis of pre-eclampsia in singleton pregnancy: implications for clinical practice. Ultrasound Obstet Gynecol 2015; 45: 241 -246 


\section{CME•thieme.de}

CME-Teilnahme

- Viel Erfolg bei lhrer CME-Teilnahme unter http://cme.thieme.de

- Diese Fortbildungseinheit ist 12 Monate online für eine CME-Teilnahme verfügbar.

- Sollten Sie Fragen zur Online-Teilnahme haben, unter

http://cme.thieme.de/hilfe finden Sie eine ausführliche Anleitung.

\section{CME-Fragen}

1 Welche Aussage ist falsch? Die nichtinvasive Chromosomentestung (NIPT) ist ...

A ein Verfahren, das mit einer hohen Genauigkeit aus dem Blut der Mutter bestimmte Chromosomenstörungen des Kindes detektieren kann.

B besonders geeignet, um eine Trisomie 21 zu detektieren.

C auch geeignet zur Detektion von Trisomie 13 und 18.

D auch bei Zwillingen gut durchführbar.

E insbesondere zur Detektion von Punktmutationen geeignet.

Welche Aussage ist richtig? Die nichtinvasive Chromosomentestung (NIPT) ...

A ist ein diagnostischer Test.

$B$ ist ein Screeningtest.

C erfordert bei Vorliegen eines positiven (auffälligen) Ergebnisses keine weitere invasive Diagnostik.

D erfordert bei Vorliegen eines negativen (unauffälligen) Ergebnisses eine invasive Diagnostik zur Bestätigung des Ergebnisses.

E ersetzt die invasive Diagnostik.

3 Welche Aussage ist richtig? Die NIPT sollte nach heutigem Konzept ...

A allen Schwangeren angeboten werden.

B allen Schwangeren angeboten werden, die älter als 35 Jahre alt sind.

C Schwangeren angeboten werden, die auffällige Befunde in der frühen Feindiagnostik haben, z. B. Fehlbildungen, die auf ein sehr hohes Risiko für Chromosomenstörungen schließen lassen.

D Frauen mit einem intermediären Risiko, im Bereich von 1:150 und 1:1000, angeboten werden.

E Frauen angeboten werden, die ein niedriges Risiko von weniger als 1:1000 haben.

\section{Welche Aussage über NIPT trifft zu?}

A Jeder Arzt kann die Beratung und Befundmitteilung bei NIPT durchführen.

B Nur Ärzte, die sich durch eine spezifische Weiterbildung für die genetische Beratung nach Gendiagnostikgesetz qualifiziert haben, dürfen über NIPT beraten.

C Eine Ultraschalluntersuchung muss bei unauffälligem NIPT-Befund nicht mehr angeboten werden.

D Alles trifft zu.

E Nichts trifft zu.

\section{Welche Aussage über die frühe Feindiagnostik im Rahmen des ETS trifft nicht zu?}

A Eine verdickte NT kann ein Hinweiszeichen für Chromosomenstörungen sein.

B Das Nasenbein, der Ductus venosus und die Trikuspidalklappe können wichtige Hinweiszeichen für Chromosomenstörungen liefern.

C Neben der „Suche“ nach Hinweiszeichen sollte die gesamte Anatomie des Feten sorgfältig betrachtet werden.

D Die Serumbiochemie kann zusätzlich die Detektionsrate erhöhen.

E Mit dem Algorithmus aus NT, zusätzlichen Markern und Serumbiochemie können Detektionsraten bis $99 \%$ erreicht werden. 
Welche Aussage über die frühe fetale Echokardiografie trifft zu?

A Das Herz muss im ETS nicht untersucht werden.

B Im ETS können die meisten Herzfehler sicher erkannt werden.

C Eine verdickte NT kann ein Hinweiszeichen für das Vorliegen eines fetalen Herzfehlers sein.

D Bei euploiden Feten ist die Nackenfalte immer im Normbereich.

E Das Flussmuster im Ductus venosus spielt keine Rolle für die Entdeckung von Herzfehlern.

7 Welche Aussage über das Screening auf Neuralrohrdefekte im ETS trifft nicht zu?

A Die Beurteilung der sog. Intracranial Translucency (IT) erfolgt in der gleichen Bildeinstellung, in der NT und Nasenbein gemessen werden.

B Die IT ersetzt die Beurteilung der Wirbelsäule in der Feindiagnostik.

C Die IT kann ein Hinweiszeichen für das Vorliegen eines Neuralrohrdefektes sein.

D Weitere Hinweiszeichen sind die Beurteilung der Breite der Cisterna magna oder der Hirnstammdurchmesser.

E In einer prospektiven Studie werden hohe Entdeckungsraten von Neuralrohrdefekten berichtet, wenn im 1. Trimester IT und CM beurteilt werden.

8 Welche Aussage über das Präeklampsiescreening im Rahmen des ETS trifft zu?

A Mittels Anamnese können nur ca. 50\% der später auftretenden Präeklampsien vorhergesagt werden.

B Die Kombination aus Anamnese und mütterlichem Blutdruck und Doppler der A. uterina erlaubt eine Vorhersage von ca. $90 \%$.

C Wenn Anamnese, Doppler, Blutdruck und die Serummarker PAPP-A und freies Beta-hCG kombiniert werden, können bis zu 96\% der frühen Präeklampsien vorhergesagt werden.

D Keine der Aussagen trifft zu.

E Alle Aussagen treffen zu.

$9 \quad$ Welche Aussage zum Präeklampsiescreening im ETS trifft nicht zu?

A Beim ETS-Algorithmus zum Präeklampsiescreening kommt prinzipiell das gleiche Vorgehen wie beim ETS auf Aneuploidien zum Einsatz.

B Die Konsequenz aus einem screeningpositiven Befund ist grundlegend von der beim Aneuploidiescreening verschieden, da es keinen diagnostischen Test auf Präeklampsie gibt.

C Das Präeklampsiescreening kann helfen, Patientinnen mit Risikokonstellation zu identifizieren.

D Das Präeklampsiescreening sollte nur Frauen mit einem ausgeprägten Risikoprofil angeboten werden.

E In die Beratung muss eine ausführliche Beratung über Limitationen und Konsequenzen des Screenings einfließen.

10 Welche Aussage zur Präeklampsieprophylaxe trifft zu?

A Metaanalysen konnten zeigen, dass eine bis zu 50\%ige Risikoreduktion möglich ist, wenn Aspirin frühzeitig (<16 SSW) gegeben wird.

B In einer prospektiv randomisierten Studie konnte gezeigt werden, dass eine bis zu 90\%ige Risikoreduktion bei früher AspirinTherapie erreicht werden kann.

C Es macht keinen Unterschied im Hinblick auf die Wirksamkeit, ob man die Aspirin-Gabe vor oder nach der 16. SSW beginnt.

D Niedermolekulares Heparin kann eine Präeklampsie effizient verhindern.

E Niedermolekulares Heparin ist Mittel der Wahl, um die Wiederholung einer frühen Präeklampsie zu vermeiden. 